\title{
SÍNDROME DE BURNOUT EM DOCENTES UNIVERSITÁRIOS DE INSTITUIÇÕES
}

\author{
PRIVADAS
}

\author{
Andrea Loly Kraft Horta Barbosa ${ }^{1}$ \\ Rute Grossi-Milani² \\ Eraldo Schunk Silva ${ }^{3}$ \\ Regiane da Silva Macuch ${ }^{4}$ \\ Lucia Elaine Ranieri Cortez ${ }^{5}$ \\ Recebido em: 12 fev. 2018 \\ Aceito em: 15 out. 2018
}

RESUMO: A jornada de trabalho extenuante e os problemas sociais experimentados pelos docentes em instituições educacionais podem torná-los vulneráveis à síndrome de Burnout. Este estudo objetivou avaliar a síndrome de Burnout em docentes universitários de instituições privadas. Foi aplicado a 141 professores um questionário referente a variáveis sociodemográficas e atividade docente, e a Escala de Caracterização do Burnout. Os resultados foram descritos aplicando-se o teste Qui-quadrado para se verificar a associação entre o grau das dimensões "Exaustão Emocional", "Desumanização" e "Decepção". Para testar o efeito das dimensões sobre as variáveis empregou-se o teste de Kruskal-Wallis, seguido do teste post-hoc de Dunn. A maioria dos docentes foi do sexo feminino, com idade abaixo de 40 anos e horistas. O tempo de atividade docente influenciou no aparecimento das dimensões exaustão, desumanização e decepção. Conclui-se que os índices apresentados pelos docentes nas dimensões sinalizam um processo de Burnout em curso. Palavras-chave: Burnout. Trabalho docente. Stress ocupacional. Qualidade de vida no trabalho.

\section{BURNOUT SYNDROME IN UNIVERSITY TEACHERS OF PRIVATE INSTITUTIONS}

ABSTRACT: The fatiguing workday and the social problems experienced by teachers in Brazilian educational institutions can make them vulnerable to Burnout syndrome. This study aimed to evaluate the Burnout Syndrome in university teachers of private institutions in the northwest and west-central state of Paraná. It was applied to 141 teachers a questionnaire regarding sociodemographic variables and teaching activities. Also used the Burnout Characterization Scale. Data were described using frequency tables, using the chi-square test to verify the association between the degree of the dimensions "Emotional Exhaustion", "Dehumanization" and "Deception". To test the effect of the dimensions of the variables we used the Kruskal-Wallis test, followed by post-hoc Dunn test and a confidence level of $95 \%(\alpha=0.05)$. The majority of teachers were female, aged below 40 years and hourly. The teaching activity of time influence the onset of dimensions Exhaustion, Dehumanization and Deception. It can be concluded that teachers have indexes as the dimensions, signaling an ongoing Burnout process.

\footnotetext{
${ }^{1}$ Centro Universitário de Maringá - UNICESUMAR.

2 Centro Universitário de Maringá - UNICESUMAR.

${ }^{3}$ Docente da Universidade Estadual de Maringá.

${ }^{4}$ Centro Universitário de Maringá - UNICESUMAR.

${ }^{5}$ Centro Universitário de Maringá - UNICESUMAR.
} 
Keywords: Burnout. Health promotion. Teaching work. Occupational stress. Quality of work life.

\section{INTRODUÇÃO}

O mundo está passando por várias mudanças tecnológicas, científicas, econômicas, políticas, culturais e sociais em prejuízo à qualidade de vida. Há um acentuado aumento do estresse nos indivíduos, comprometendo sua saúde e bem-estar, além de efeitos importantes no cenário do trabalho (DA PAZ, 2014).

O trabalho sofreu muitas transformações nas últimas décadas e as diversas profissões, bem como a carreira docente, continuam passando por profundas mudanças, desde contratos flexíveis, aumento da carga horária e exigência de diversidade no trabalho, o que repercute na qualidade de vida profissional e saúde dos docentes (COUTINHO; LISBÔA, 2011).

A jornada de trabalho extenuante e os problemas sociais experimentados pelos docentes em suas instituições e salas de aula, adicionados ao acirrado processo brasileiro de desvalorização salarial contribui para o crescente adoecimento destes profissionais. Além disso, os educadores em instituições de ensino superior conciliam atividades pedagógicas, como pesquisa e extensão, atendendo questões relacionadas à produção científica, além de ter tarefas administrativas (VIEIRA et al., 2010).

As universidades estão em um contexto de evolução e globalização, o cenário do trabalho docente tem mudado constantemente, com aumento das exigências em relação ao profissional. As instituições buscam docentes qualificados e polivalentes para exercerem funções de acordo com suas demandas específicas, podendo estes profissionais ficarem vulneráveis e desenvolverem a síndrome de Burnout (ANDRADE; CARDOSO, 2012). Conforme Zambon (2014), o Burnout é o resultado de uma resposta emocional a uma situação de estresse crônico e prolongado no trabalho de profissionais, que têm como função o contato com outras pessoas.

Em relação aos fatores profissionais, os trabalhadores que desenvolvem a síndrome de Burnout apresentam grandes expectativas em relação ao seu desenvolvimento e desempenho, mas, em função de diferentes obstáculos, não alcançaram o retorno esperado. O nível de expectativa é drasticamente oposto à realidade, e estes profissionais que persistem em tentar alcançá-lo, tornam suas trajetórias tempestuosas e problemáticas, levando à diminuição de recursos individuais, comprometendo suas habilidades (ZAMBON, 2014). Burnout é o sofrimento vivenciado pelo educador diante deste impasse, entre o que pode realmente realizar e o que efetivamente consegue fazer. Este dilema é a raiz de suas aflições e dor (SILVA, 2014).

A síndrome de Burnout ou síndrome do esgotamento profissional é constituída por três elementos centrais: exaustão emocional, despersonalização e diminuição do envolvimento pessoal no trabalho (MASLACH; SCHAUFELI; LEITER, 2001). Apesar dos 
estudos acerca da síndrome de Burnout, no que se refere a sua avaliação, ainda se constitui uma situação de dificuldade para os pesquisadores, pois requerem estudos de validação e de adaptação dos instrumentos de acordo com a realidade da população a ser estudada. No Brasil, alguns autores adaptaram e validaram o Maslach Burnout Inventory (MBI), como Lautert (1997) e Tamayo (2003). No entanto, depararam-se com índices baixos de consistência interna para determinados fatores. A Escala de Caracterização do Burnout (ECB) foi desenvolvida para o uso com diferentes profissionais, como os de saúde, professores e policiais. É uma escala adaptada à cultura brasileira e seus fatores apresentam qualidades psicométricas superiores a outras versões brasileiras do $\mathrm{MBI}$ (TAMAYO; TRÓCCOLI, 2009).

Desde os primeiros estudos sobre a síndrome de Burnout, a categoria docente tem sido uma das mais investigadas (CARLOTTO, 2011) e a prevalência desta síndrome na classe do professorado é alta, podendo interferir no processo de ensino-aprendizagem (SILVEIRA et al., 2014). Diante desta problemática, este trabalho objetiva avaliar a síndrome em docentes de instituições privadas de ensino superior.

\section{MÉTODO}

\section{PARTICIPANTES}

A pesquisa transcorreu em quatro instituições privadas de ensino superior do noroeste e centro-oeste do Paraná. A amostra inicial foi de 359 docentes, mas participaram da pesquisa efetivamente 141 docentes de ambos os sexos. Foram excluídos professores que exercem somente atividades administrativas, docentes substitutos, e aqueles que estão nas instituições há menos de seis meses.

\section{INSTRUMENTO}

Foi utilizado um questionário autoaplicado sobre dados sociodemográficos (idade, sexo, renda, estado civil), acrescido de informações sobre aspectos profissionais (carga horária de trabalho, quantidade de alunos por sala e para orientação, vínculo empregatício, tempo de exercício de profissão). O instrumento aplicado para avaliar a síndrome de Burnout foi a ECB (TAMAYO; TRÓCCOLI, 2009).

A ECB é autoaplicada e analisa como o sujeito vivencia seu trabalho, de acordo com as três dimensões estabelecidas pelo modelo teórico de Burnout, proposto por Maslach. Neste instrumento, os autores nomearam os fatores componentes de Burnout de modo levemente diverso do que é frequentemente encontrado em estudos empíricos. Entretanto, as definições são muito próximas e representam o construto com índices 
fidedignos no contexto brasileiro. A escala é constituída de 35 itens, distribuídos em três dimensões, com uma escala de resposta de 5 pontos: nunca (1) raramente (2) algumas vezes (3) frequentemente (4) e sempre (5). A primeira dimensão (exaustão emocional) agrupa 12 itens que transmitem a ideia de esgotamento, cansaço e desgaste no trabalho. A segunda (desumanização) agrupa dez itens que sugerem dureza emocional, desinteresse e atitudes negativas no trato com os usuários de seus serviços. E, por fim, a terceira dimensão do Burnout (decepção), que denota desânimo, desespero, frustração e inadequação no trabalho, que agrupa 13 itens.

\section{PROCEDIMENTOS}

Primeiramente, os professores foram abordados sobre o motivo do estudo e convidados a participar da pesquisa. Os docentes que concordaram foram esclarecidos de que esta pesquisa não apresentava finalidade avaliativa individual e ou institucional e que suas identidades seriam mantidas em sigilo e no anonimato. A coleta de dados aconteceu entre os meses de março a maio de 2015, após a aprovação do Comitê de Ética em Pesquisa, sob parecer ํㅜ 006685/2015. Os docentes foram entrevistados na sala dos professores, por ordem de chegada após assinatura do termo de consentimento livre esclarecido (TCLE).

Os dados foram descritos por meio de tabelas de frequência simples e cruzadas. Para a avaliação do grau de linearidade entre as dimensões "Exaustão Emocional", "Desumanização" e "Decepção" e as variáveis idade, tempo de exercício da profissão, tempo de formação, número de vínculos, número de alunos, carga horária dedicada às atividades de ensino, carga horária dedicada às outras ocupações profissionais e carga horária dedicada ao preparo de avaliações foi utilizado o coeficiente de correlação de Spearman $(\rho)$. Foi utilizado o teste Qui-quadrado para verificar a associação entre o grau de agravamento das dimensões "Exaustão Emocional", "Desumanização" e "Decepção" e as demais variáveis categóricas. Para testar o efeito das dimensões sobre as variáveis citadas anteriormente, foi utilizado o teste de Kruskal-Wallis, seguido do teste post-hoc de Dunn. Os dados foram analisados no programa SAS versão 9.3. Adotou-se um nível de confiança de $95 \%(\alpha=0,05)$.

\section{RESULTADOS}

A maioria dos docentes ( $n=78,55,32 \%)$ entrevistada é do sexo feminino e casada $(\mathrm{n}=88,62,41 \%)$. Quanto à idade, $75,18 \%(\mathrm{n}=106)$ está abaixo de 40 anos. Em relação ao grau de titulação, a maior parte destes é mestre $(n=79,56,03 \%)$, seguida de especialistas $(n=45,31,91 \%)$ e doutores $(n=17,12,06 \%)$.

Em relação à atividade docente (tabela 1), grande parte é horista $(n=67,47,52 \%)$, com menos de dez anos de docência $(n=95,67,86 \%)$ e com faixa salarial abaixo de $R \$$ 
$2.400,00$ (n=80, 56,74\%). Quanto ao número de vínculos empregatícios, 45,39\%, 39,72\% e $14,89 \%$ correspondem a dois, um e três respectivamente.

Pode-se observar o predomínio (43,97\%) de docentes com carga horária semanal de $40 \mathrm{~h}$ ou mais de trabalho no ensino. Em relação à quantidade de alunos por sala, apenas 12 (8,51\%) responderam atender abaixo de 20 , sendo que $65 \%$ trabalham com mais de 30 alunos. Quanto à atividade de orientação, a maioria (70,21\%) o faz acima de três alunos.

Tabela 1 - Dados relativos à área de atividade profissional dos professores universitários de instituições privadas nas regiões noroeste e centro-oeste do PR, 2015.

\begin{tabular}{|c|c|c|}
\hline & $\mathbf{n}$ & $\%$ \\
\hline \multicolumn{3}{|l|}{ Quantidade de alunos por sala } \\
\hline Até 20 alunos & 12 & 8,51 \\
\hline De 21 a 30 alunos & 37 & 26,24 \\
\hline De 31 a 40 alunos & 45 & 31,91 \\
\hline Mais de 40 alunos & 47 & 33,33 \\
\hline \multicolumn{3}{|c|}{ Quantidade de alunos para orientação } \\
\hline Nenhum & 19 & 13,48 \\
\hline Até dois & 23 & 16,31 \\
\hline Três ou quatro & 35 & 24,82 \\
\hline Cinco ou seis & 34 & 24,11 \\
\hline Mais de seis & 30 & 21,28 \\
\hline \multicolumn{3}{|l|}{ Tipo de vínculo empregatício } \\
\hline Horista & 67 & 47,52 \\
\hline T20 & 34 & 24,11 \\
\hline T40 & 40 & 28,37 \\
\hline \multicolumn{3}{|l|}{ Faixa salarial (R\$) } \\
\hline Até 1200,00 & 31 & 21,99 \\
\hline De 1200,00 a 2400,00 & 49 & 34,75 \\
\hline De 2400,00 a 5000,00 & 61 & 43,26 \\
\hline \multicolumn{3}{|c|}{ Há quanto tempo você exerce a profissão de docente } \\
\hline Menos de 10 anos & 95 & 67,86 \\
\hline Mais de 10 anos & 45 & 32,14 \\
\hline \multicolumn{3}{|c|}{ Quantos vínculos empregatícios você tem } \\
\hline Um & 56 & 39,72 \\
\hline Dois & 64 & 45,39 \\
\hline Três & 21 & 14,89 \\
\hline \multicolumn{3}{|c|}{$\begin{array}{l}\text { Qual a carga horária semanal que você se dedica ao } \\
\text { ensino }\end{array}$} \\
\hline Menos de 20 horas semanais & 29 & \\
\hline De 20 a 39 horas semanais & 50 & 35,46 \\
\hline 40 horas semanais ou mais & 62 & 43,97 \\
\hline
\end{tabular}

FONTE: Dados da pesquisa.

A tabela 2 mostra os níveis da síndrome de Burnout, segundo as três dimensões: exaustão, desumanização e decepção.

Quanto aos níveis de Burnout, nas três dimensões, observa-se que 11 (7,80\%) docentes entrevistados se encontram em nível leve, 26 (18,44\%) em nível moderado e 12 $(8,51 \%)$ entrevistados em nível alto nas três dimensões (exaustão, desumanização e decepção). É preocupante imaginar que os docentes que se encontram no nível moderado possam evoluir para o nível alto, ou seja, o que representaria em torno de $27 \%$ do grupo estudado. 
Tabela 2 - Nível de desumanização, exaustão e decepção dos professores universitários de instituições privadas nas regiões noroeste e centro-oeste do PR, 2015.

\begin{tabular}{|c|c|c|c|c|c|c|}
\hline \multirow[t]{3}{*}{ Exaustão } & & & \multicolumn{4}{|c|}{ DESUMANIZAÇÃO } \\
\hline & & & Leve & Moderado & Alto & Total \\
\hline & & & $\mathrm{n}$ & $\mathbf{n}$ & $\mathbf{n}$ & $\mathrm{n}$ \\
\hline \multirow{4}{*}{ Leve } & \multirow{4}{*}{ DECEPÇÃO } & Leve & 11 & 4 & 2 & 16 \\
\hline & & Moderado & 5 & 5 & 1 & 11 \\
\hline & & Alto & 0 & 0 & 0 & 0 \\
\hline & & Total & 16 & 9 & 3 & 28 \\
\hline \multirow{4}{*}{ Moderado } & \multirow{4}{*}{ DECEPÇÃO } & Leve & 2 & 11 & 0 & 13 \\
\hline & & Moderado & 6 & 26 & 12 & 44 \\
\hline & & Alto & 2 & 10 & 8 & 20 \\
\hline & & Total & 10 & 47 & 20 & 77 \\
\hline \multirow{4}{*}{ Alto } & \multirow{4}{*}{ DECEPÇÃO } & Leve & 0 & 0 & 0 & 0 \\
\hline & & Moderado & 4 & 9 & 2 & 15 \\
\hline & & Alto & 1 & 8 & 12 & 21 \\
\hline & & Total & 5 & 17 & 14 & 36 \\
\hline
\end{tabular}

FONTE: Dados da pesquisa.

Na tabela 3 é apresentada comparação entre as dimensões exaustão, decepção e desumanização e a variável tempo de docência.

Tabela 3 - Teste de kruskal-wallis, seguido do teste de comparações múltiplas post hoc de dunn, comparando as dimensões "decepção, exaustão e desumanização" para os professores universitários de instituições privadas nas regiões noroeste e centro-oeste do PR, 2015.

\section{EXAUSTÃO}

\begin{tabular}{|c|c|c|c|c|}
\hline Há quanto tempo você exerce a profissão de docente & $\mathbf{n}$ & média & $\mathbf{F}$ & p-valor \\
\hline Menos de 5 anos & 75 & $74,01^{a}$ & & \\
\hline De 5 a 9 anos & 42 & $71,00^{\mathrm{a}}$ & 4,53 & $0,0125^{*}$ \\
\hline 10 anos ou mais & 24 & $46,58^{\mathrm{b}}$ & & \\
\hline \multicolumn{5}{|l|}{ DESUMANIZAÇÃO } \\
\hline Há quanto tempo você exerce a profissão de docente & $\mathbf{n}$ & média & $\mathbf{F}$ & p-valor \\
\hline Menos de 5 anos & 75 & $68,75^{a}$ & & \\
\hline De 5 a 9 anos & 42 & $61,24^{a}$ & 4,53 & $0,0125^{*}$ \\
\hline 10 anos ou mais & 24 & $72,29 b$ & & \\
\hline DECEPÇÃO & $\mathbf{n}$ & Média & $\mathbf{F}$ & p-valor \\
\hline \multicolumn{5}{|l|}{ Há quanto tempo você exerce a profissão de docente } \\
\hline Menos de 5 anos & 75 & $73,52^{a}$ & & \\
\hline De 5 a 9 anos & 42 & $63,59^{a}$ & 4,22 & $0,0167^{*}$ \\
\hline 10 anos ou mais & 24 & $47,12^{\mathrm{b}}$ & & \\
\hline
\end{tabular}

*significativo em nível de 95\% de confiança $(\alpha=0,05)$. Os números marcados com a mesma letra não diferem entre si pelo teste de Kruskal-Wallis, seguido do teste de Dunn.

FONTE: Dados da pesquisa.

É visto que em todos os níveis não se verifica diferença estatística quanto a menos de cinco anos e cinco a nove anos de atividade docente. No entanto, em todas as três dimensões há diferença significativa quanto ao tempo da atividade docente de dez anos ou mais e abaixo deste. Em relação aos níveis exaustão e decepção, estes são mais evidentes em docentes abaixo de dez anos do exercício da profissão; já a dimensão desumanização, é mais expressiva em docentes com maior tempo de docência.

$\mathrm{Na}$ tabela 4 é apresentada a associação significativa entre o tempo de atividade docente e as dimensões exaustão e desumanização.

Tabela 4 - Variáveis associadas (teste qui-quadrado) à dimensão "exaustão e desumanização" para os 
professores universitários de instituições privadas nas regiões noroeste e centro-oeste do PR, 2015.

\begin{tabular}{|c|c|c|c|c|c|}
\hline \multirow{3}{*}{ Exaustão } & \multicolumn{4}{|c|}{ Há quanto tempo você exerce a profissão de docente } & \multirow{6}{*}{$0,0123^{*}$} \\
\hline & \multicolumn{2}{|c|}{ Menos de 10 anos } & \multicolumn{2}{|c|}{10 anos ou mais } & \\
\hline & $\mathbf{n}$ & $\%$ & $\mathbf{n}$ & $\%$ & \\
\hline Alta & 30 & 25.74 & 12 & 50.00 & \\
\hline Moderada & 62 & 52,99 & 10 & 41,67 & \\
\hline Baixa & 25 & 21,37 & 2 & 8,33 & \\
\hline \multirow{3}{*}{ Desumanização } & \multicolumn{4}{|c|}{ Há quanto tempo você exerce a profissão de docente } & \multirow{6}{*}{$0,0062^{*}$} \\
\hline & \multicolumn{2}{|c|}{ Menos de 10 anos } & \multicolumn{2}{|c|}{10 anos ou mais } & \\
\hline & n & $\%$ & $\mathbf{n}$ & $\%$ & \\
\hline Alta & 30 & 25,74 & 10 & 50,00 & \\
\hline Moderada & 62 & 52,99 & 10 & 50,00 & \\
\hline Baixa & 25 & 21,37 & 0 & 0,00 & \\
\hline
\end{tabular}

*significativo em nível de $95 \%$ de confiança $(\alpha=0,05)$.

FONTE: Dados da pesquisa.

Pode-se observar a correlação positiva e significativa entre o tempo de docência e as duas dimensões de Burnout, evidenciando que o maior tempo dedicado à docência favorece o aumento do aparecimento de sintomatologia associada ao Burnout. Verifica-se nível de 20,19\% para docentes com menos de dez anos de exercício profissional. No entanto, os valores aumentam para $50 \%$ em docentes com dez anos ou mais de exercício profissional. Resultados mais preocupantes são observados para a dimensão desumanização, uma vez que professores com tempo de atividade docente com dez ou mais anos, não se encontram mais no nível baixo, ou seja, estão entre os níveis moderado $(50 \%)$ e alto $(50 \%)$.

\section{DISCUSSÃO}

O perfil da nossa população de estudo mostrou que a carga horária de um docente não se resume apenas às 40 horas semanais, na maioria das vezes ele tem que trabalhar além desta carga horária, pois nas instituições privadas predominam os "horistas", o que sinaliza uma realidade profissional com grande demanda de trabalho. Esta sobrecarga reduz o tempo de lazer destes profissionais, com consequente propensão para 0 desequilíbrio na saúde mental. Somado a isso, o dia a dia dos docentes que atuam no ensino superior exige uma busca cada vez maior por produtividade e qualidade do professorado, o que pode gerar uma exposição favorável para o estresse e, muitas vezes, para o Burnout (PAIVA; GOMES; HELAL, 2015).

Esteves (2013) relata que professores com atividade docente o tempo inteiro sem exclusividade apresentam maior nível de exaustão emocional. A autora afirma que a necessidade de os professores trabalharem em mais de uma instituição, aumenta sua sobrecarga de trabalho e, reduz seu tempo de lazer, com consequente propensão para 0 desequilíbrio na saúde mental.

A análise quanto às dimensões mostrou que os docentes se encontram em nível 
significativo nas três dimensões de Burnout, sendo mais expressivo o nível moderado. Souza et al. (2013) identificaram, em estudo sobre Burnout, manifestações psíquicas, comportamentais e defensivas como negligência, irritabilidade, tendência ao isolamento, sentimento de onipotência. Cabe salientar que tais sintomas dependem de características pessoais de cada um e do ambiente de trabalho. Ainda que o docente apresente sentimentos e atitudes das dimensões, é complicado admitir esses comportamentos, como não tratar seus alunos com simpatia e afeição ou cogitar a probabilidade de que a docência não o realiza profissionalmente.

O professor exerce papel fundamental, pois dele dependem os discentes que estão a seu encargo para desenvolverem a aprendizagem. O professor e o seu labor são muito importantes para a educação e sua saúde é um aspecto que pode colocar sua eficiência em risco (CRUZ; MATOS; DINIZ, 2016). Os alunos necessitam de docentes, que sejam zelosos, acolhedores e bons profissionais, pois este aspecto é fundamental para seu desenvolvimento (BULLOUGH, 2015). Entretanto, o sistema de ensino exige muito em termos emocionais, o que pode explicar o esgotamento dos docentes (TUXFORD; BRADLEY, 2014). O profissional com Burnout pode interferir na aprendizagem, no comportamento e na motivação dos alunos, tendo consequências não somente no campo pessoal-profissional, mas também no processo educacional.

Neste estudo, a dimensão desumanização mostrou-se expressiva em docentes com maior tempo de docência. Conforme Benevides-Pereira (2012), a desumanização é um elemento fundamental do diagnóstico da síndrome de Burnout, que a distingue do estresse, da depressão e de outros transtornos. Schuster, Dias e Battistella (2015) relatam que na dimensão desumanização ou despersonalização há sentimento de indiferença ou uma atitude de distanciamento e de cinismo para com os alunos, colegas e a instituição.

Os participantes deste estudo se encontram nas três dimensões de Burnout e percebemos que os mais inexperientes estão com média maior em exaustão e decepção e os mais experientes em desumanização. No entanto, estes profissionais ainda não estão com decepção ou baixa realização profissional, talvez isso possa ser explicado pela afirmação de que estes docentes apesar de já estarem com sintomas de Burnout, ainda não entraram na última fase da síndrome. Possivelmente, tenham adotado mecanismos de enfrentamento dos sintomas do Burnout e resiliência, conforme Souza e Almeida (2009) abordam em artigo sobre o tema resiliência e referem que pessoas resilientes são aquelas que conseguem enfrentar situações traumáticas, superando-as e retomando o seu desenvolvimento, saindo-se delas fortalecidas.

Entretanto, não se encontra concordância na literatura, pois, enquanto alguns autores descrevem o Burnout como um processo de desgaste que se desenvolve ao longo do tempo de trabalho, outros autores têm apontado maior incidência nos que ingressam no mercado de trabalho, possivelmente pela pouca experiência na profissão, por não haver ainda desenvolvido formas de enfrentamento adequadas às situações ou, ainda, por fatores associados à pouca idade, sendo referidos os valores mais altos de Burnout que ocorrem entre dez e 15 anos de docência (CODO; VASQUEZ-MENEZES, 1999). 
Silva e Carlotto (2003) referem que quanto maior o tempo de atividade docente aumentam também sintomas de desgaste, possivelmente pelo grande comprometimento e exigências sociais em relação às responsabilidades e à função docente. Esta afirmação corrobora com os resultados do nosso estudo.

Segundo Maslach, Schaufeli e Leiter (2001), a exaustão emocional é o principal fator que caracteriza a síndrome de Burnout, sendo condição necessária, mas não suficiente para diagnosticá-la. Indispensável é, portanto, que o indivíduo apresente outros sintomas, tais como despersonalização (desumanização) e baixa realização profissional (decepção no trabalho) para que a síndrome fique caracterizada.

\section{CONSIDERAÇÕES FINAIS}

Neste estudo, os profissionais se mostraram tão exaustos, desumanos, quanto decepcionados, o que pode prejudicar consideravelmente a qualificação e o relacionamento destes com alunos e colegas. É fundamental salientar que a prevenção e a promoção da saúde do docente é uma tarefa conjunta entre o professorado, discentes, instituição de ensino e sociedade, o que requer reflexões e ações que conduzam a mudanças desta realidade.

Os docentes mostraram índices moderados quanto às dimensões exaustão, desumanização e decepção emocional, sinalizando o processo de Burnout em curso. Na análise dos dados, verificou-se correlação positiva entre o maior tempo de trabalho docente e o aparecimento de sintomatologia associada ao Burnout, evidenciando as dimensões exaustão e decepção nos mais inexperientes e a dimensão desumanização nos professores com maior tempo de docência.

Ressalta-se a importância da realização de novas pesquisas mais abrangentes em relação à amostra de docentes de ensino superior, tanto de instituições privadas e públicas, assim como outros delineamentos e variáveis para aumentar o conhecimento sobre o Burnout, bem como estratégias de enfrentamento para serem utilizadas por estes professores, favorecendo, desta forma, a promoção da saúde docente.

\section{REFERÊNCIAS}

ANDRADE, P. S. de; OLIVEIRA CARDOSO, T. A. de. Prazer e dor na docência: revisão bibliográfica sobre a Síndrome de Burnout. Saúde e Sociedade, v.21, n. 1, p. 129-140, 2012. DOI:10.1590/S0104-12902012000100013

BENEVIDES-PEREIRA, A. M. T. Considerações sobre a síndrome de burnout e seu impacto no ensino. Boletim de Psicologia, v.62, n.137, p.155-168, 2012. Disponível em: http://pepsic.bvsalud.org/scielo.php?script=sci_arttext\&pid=S0006$59432012000200005 \&$ lng=pt\&tlng=pt. 
BULLOUGH, R. V. Differences? Similarities? Male teacher, female teacher: An instrumental case study of teaching in a Head Start classroom. Teaching and Teacher Education, v.47, p.13-21, 2015. DOI: http://dx.doi.org/10.1016/j.tate.2014.12.001

CARLOTTO, M. S. Síndrome de Burnout em professores: prevalência e fatores associados. Psicologia: Teoria e Pesquisa, v. 27, n.4, p.403-410, 2011. DOl: http://dx.doi.org/10.1590/S0102-37722011000400003.

CODO, W.; VASQUES-MENEZES, I. O que é Burnout? In: W. CODO (Coord.). Educação e carinho. (pp. 237 - 254). São Paulo: Vozes, 1999.

COUTINHO, C. P.; LISBÔA, E. S. Sociedade da informação, do conhecimento e da aprendizagem: desafios para educação no século XXI. Revista de Educação, v. 18, n.1, p. 5-22, 2011. Disponível em: http://hdl.handle.net/1822/14854.

CRUZ, J.; de MATOS, M. G.; DINIZ, J. A. A relação professor-aluno: um olhar necessário para o contexto. Revista de Psicologia da Criança e do Adolescente, v.6, n.2, p.145154, 2016. Disponível em:

http://revistas.lis.ulusiada.pt/index.php/rpca/article/view/2316/2438

DA PAZ, M. P. O estresse no cotidiano de professores da Educação Superior. Revista FSA (Faculdade Santo Agostinho), Teresina, v.6, n.1, p. 66-77, 2014. Disponível em: file:///D:/Downloads/425-1026-1-PB.pdf

ESTEVES, A. M. A. O Burnout e o engagement: estudo numa amostra de professores do ensino superior público português. 2013. $82 \mathrm{f}$. Dissertação (Mestrado em Gestão das Organizações - área de Gestão de Empresas) - Curso de Mestrado em Gestão das Organizações. ISCAP, Instituto Superior de Contabilidade e Administração do Porto, Porto.

LAUTERT, L. The professional fatigue: empirical study with hospital nurses. Revista Gaúcha de Enfermagem, Porto Alegre, v.18, n.2, p. 133-144, 1997.

MASLACH, C.; SCHAUFELI, W. B.; LEITER, M. P. Job Burnout. Annual Review of Psychology, Palo Alto, v.52, n.1, p. 397-422, 2001. DOI: 10.1146/annurev.psych.52.1.397

PAIVA, K. C. M. D.; GOMES, M. Â. D. N.; HELAL, D. H. Estresse ocupacional e síndrome de burnout: proposição de um modelo integrativo e perspectivas de pesquisa junto a docentes do ensino superior. Gestão \& Planejamento-G\&P, v. 16, n.3, 2015. Disponível em: http://www.revistas.unifacs.br/index.php/rgb/article/view/3570.

SILVA SCHUSTER, M. da; VEIGA DIAS, V. da; BATTISTELLA, L. F. Maslach Burnout Inventory-General Survey (MBI-GS): Aplicação em Universidade Público Federal. Revista da Faculdade de Administração e Economia, v.6, n.2, p. 182-195, 2015. DOI: http://dx.doi.org/10.15603/2176-9583/refae.v6n2p182-195

SILVA, G. N. da; CARLOTTO, M. S. Síndrome de Burnout: um estudo com professores da rede pública. ABRAPEE, v.7, n.2, p.145-153, 2003. Disponível em:

http://www.scielo.br/pdf/pee/v7n2/n2a04. DOI: https://doi.org/10.1590/s141385572003000200004

SILVA, M. P. G. O. da. A silenciosa doença do professor: Burnout, ou o mal-estar 
docente. Revista Científica Integrada, Guarujá, v.1, n.2, p. 1-10, 2014. Disponível em: http://www.unaerp.br/revista-cientifica-integrada/edicoes-anteriores/edicao-n-2-20141/1464-161-454-1-sm/file

SILVEIRA, K. A. et al. Indicators of stress and coping in the context of inclusive education. Educação e Pesquisa, v.40, n.1, p.127-142, 2014. DOI: http://dx.doi.org/10.1590/S151797022014000100009

SOUZA, F. das C. S.; ALMEIDA, M. C. X. de. Resiliência e educação. Revista Educação em Questão, Natal, v.35, n.21, p. 239-243, 2009. Disponível em:

https://periodicos.ufrn.br/educacaoemquestao/article/view/3963/3230

SOUZA, W. L. Conhecimento publicado acerca do absenteísmo relacionado à Síndrome de Bournout com os profissionais de enfermagem: revisão integrativa. Caderno de Graduação-Ciências Biológicas e da Saúde-UNIT/AL, v.1, n.2, p. 121-134, 2013. Disponível em: https://periodicos.set.edu.br/index.php/fitsbiosaude/article/view/637/372

TAMAYO, M. R. Validação do inventário de Burnout de Maslach. In: III CONGRESSO NORTE-NORDESTE DE PSICOLOGIA, 3, 2003, João Pessoa. Resumo... João Pessoa: UFPB, 2003. p. 392-393.

TAMAYO, M. R.; TRÓCCOLI, B. T. Construção e validação fatorial da Escala de Caracterização do Burnout (ECB). Estudos de Psicologia, Natal, v.14, n.3, p. 213-221, 2009. DOI: https://doi.org/10.1590/S1413-294X2009000300005

TUXFORD, L. M.; BRADDLEY, G. L. Emotional job demands and emotional exhaustion in teachers. Educational Psychology, [S.I.], v.66, n.1, p. 1-19, 2014. DOI:

http://dx.doi.org/10.1080/01443410.2014.912260

VIEIRA, J. S. Constituição das doenças da docência. Cadernos de Educação, Pelotas, v.37, p. 303-324, 2010. Disponível em:

http://repositorio.ufpel.edu.br/bitstream/123456789/714/3/Constitui\%C3\%A7\%C3\%A30\%2 0das\%20doencas\%20da\%20docencia.pdf

ZAMBON, E. Estratégias de prevenção ao estresse ocupacional de professores do ensino superior privado. 2014. 130 f. Tese (Doutorado em Educação) - Programa de Pós-Graduação em Educação. PUCRS, Pontifícia Universidade Católica do Rio Grande do Sul, Porto Alegre. 